\title{
Visualisation of two-dimensional kinematic data from bimanual control of a commercial gaming system used in post-stroke rehabilitation
}

\author{
Bulmaro A. Valdés, Navid Shirzad, Chai-Ting Hung, \\ H.F. Machiel Van der Loos \\ Department of Mechanical Engineering \\ The University of British Columbia \\ Vancouver, Canada \\ bulmaro.valdes@alumni.ubc.ca, \\ navids@interchange.ubc.ca, \\ tina.ct.hung@alumni.ubc.ca, \\ vdl@mech.ubc.ca
}

\begin{abstract}
Kinematic data from two stroke participants and a healthy control were collected using a novel bimanual rehabilitation system. The system employs two customized PlayStation Move Controllers and an Eye camera to track the participants' hand movements. In this study, the participants played a Facebook game by symmetrically moving both hands to control the computer's mouse cursor. The collected data were recorded during one game session, and movement distribution analysis was performed to create density plots of each participant's hand motion in the XY plane. This type of kinematic information that can be gathered by rehabilitation systems with motion tracking capabilities has the potential to be used by therapists to monitor and guide home-based rehabilitation programs.
\end{abstract}

Keywords-virtual rehabilitation; stroke therapy; social media; motion tracking; compensation; rehabilitation technology; bimanual therapy; kinematic plot.

\section{INTRODUCTION}

Commercially available gaming systems are increasingly being employed as stroke rehabilitation tools to provide opportunities for engaging repetitive practice during motor retraining [1]. Emerging research lends support to the feasibility and potential for improved upper limb functional outcomes for the stroke population [2], [3]. Because of its accessibility in the home, this type of therapy holds particular promise as an intervention when access to other treatment options is limited [2]. Reports of successful home therapy using motion-based game controllers [4], [1] include limited information regarding the utility of the extractable kinematic data afforded by these systems, as research has focused primarily on determining their motion tracking accuracy and on evaluating client outcomes by means of standardized clinical assessments [5].

The FEATHERS (Functional Engagement in Assisted Therapy through Exercise Robotics) Project aims to provide an

\author{
Stephanie M.N. Glegg \\ Therapy Department \\ Sunny Hill Health Centre for Children \\ Vancouver, Canada \\ sglegg@cw.bc.ca \\ Erin Reeds \\ Physical Therapy \\ Abilities Neurological Rehabilitation \\ Surrey, Canada \\ erin@abilitiesrehabilitation.com
}

engaging solution for physical therapy by combining bilateral movement therapy with a social media platform and computer games [6]. The target users are adults with stroke and adolescents with cerebral palsy or acquired brain injury involving hemiparesis. This paper details the kinematic data analysis of one healthy control and two stroke participants using adapted PlayStation technology as a feasibility study, and describes the potential utility of these analyses for therapists monitoring game-based home treatment programs.

\section{METHODS}

Participants were recruited from a private practice neurological clinic, where user testing was carried out to investigate the usability of the FEATHERS system. Research Ethics Board approval for this study was obtained from the University of British Columbia, along with informed consent/assent from participants and a parent/guardian or caregiver as applicable.

\section{A. System Description}

The FEATHERS system consists of three main components: FEATHERS Motion, a software application that allows the user to control the computer cursor using bilateral upper-body movements; motion tracking technologies that record the kinematics data of the users; and FEATHERS Play, a Facebook $($ application that links to numerous online games and serves as an online community for users, i.e., rehabilitation professionals and therapy clients (see [6], [7] for details).

Bilateral algorithms developed in Windows ${ }^{\circledR} 7$ were integrated with the Sony PlayStation ${ }^{\circledR}$ Move system. This system consists of the PlayStation Eye camera, which tracks the spatial locations of the PlayStation Move controllers. FEATHERS Controllers (Fig. 1) were designed to improve the ergonomic aspects of the original PlayStation Move controllers

This research is supported by the Peter Wall Solutions Initiative, NSERC (Natural Sciences and Engineering Research Council of Canada), and CONACYT (Consejo Nacional de Ciencia y Tecnología, México) 


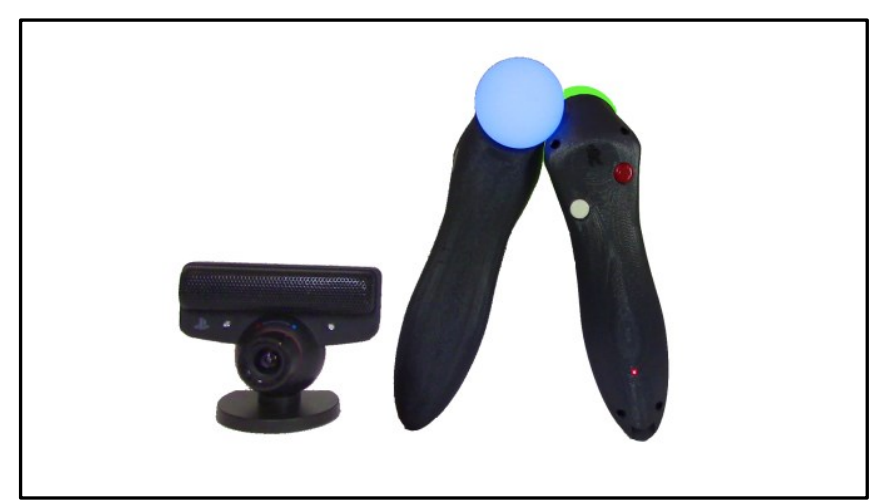

Fig. 1. FEATHERS Controllers and PlayStation Eye camera.

for the target users (e.g., minimizing the number of buttons to reduce cognitive load, and adding a tapered base for easier positioning into the hand in cases of high muscle tone) [8].

In the FEATHERS system, real-time software implements a "Visual Symmetry" (VS) mode that requires the users to control the computer cursor by moving both hands at the same time in the same direction. If the user fails to move both hands in symmetry, the computer cursor will not move. Specifically, the displacements of both hands are compared at every time step to ensure that the hand motion with the least movement in the user's frontal plane is mapped into cursor motion. This mapping mode was implemented to promote bimanual motion in the absence of therapist supervision, which prevents users from compensating by only using their unaffected arm to operate the system.

\section{B. Study Procedures}

Each participant took part in an audio- and video-recorded 90-minute session in which they were asked to complete a set of tasks to gain familiarity with the FEATHERS system and to play "Lucky Pirate" (OUAT Entertainment) using each motion tracking interface. This game (Fig. 2) provides a virtual realitybased motor exploration task set on a static background and requires players to explore the entire game screen to select and click on treasure chests that may contain gold coins. To promote engagement, the game provides visual and auditory feedback when the user opens a new treasure chest or completes a level. In addition, the user can obtain special items from the chests to extend game play. Completion of a level does not carry a time restriction; as a result users are able to progress at their own pace.

The FEATHERS Motion application allows the therapist to adjust the sensitivity of the cursor mapping to accommodate each user's range of motion. Using this feature, the system was calibrated so that each participant was capable of moving the cursor from the left to the right edges of the screen using their own bimanual movement capacity. This calibrating procedure was employed to ensure that all participants were capable of playing the game regardless of their level of function. Only game play data using the PlayStation Move interface in VS mode were of interest to the current analysis.

\section{Participants}

Subject 1 (S1) was a right-hand-dominant, 72-year-old female who had suffered a haemorrhagic stroke 49 months prior

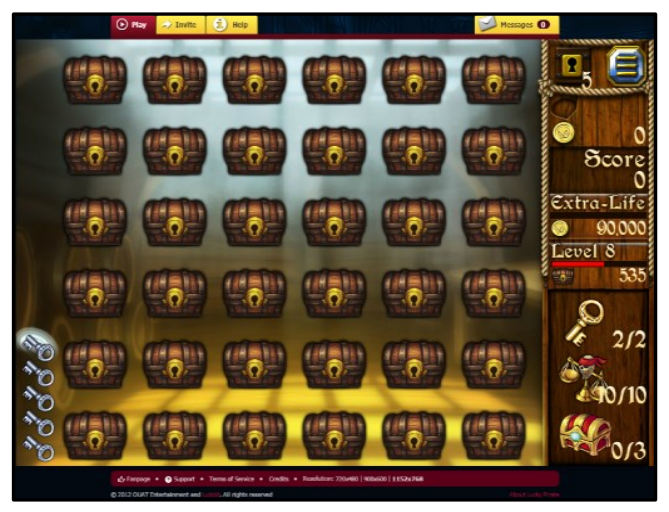

Fig. 2. Lucky Pirate Game

to this study, and presented with left hemiparesis. She was limited in the last $20^{\circ}$ of passive shoulder flexion and abduction, and presented with stiffness in retraction and upward rotation of the scapula. Her active shoulder flexion was limited to $110^{\circ}$ with significant compensatory movement patterns of side trunk flexion and shoulder elevation. While she demonstrated increased flexor tone of the arm and forearm, full passive range of motion (ROM) was observed at the elbow, but only $20^{\circ}$ of wrist extension. She was limited to $20^{\circ}$ of extension at the metacarpophalangeal (MCP) joints. Her decreased grasp strength, fine motor control and poor in-hand manipulation led her to use her left arm primarily as a helper hand.

Subject 2 (S2) was a right-hand-dominant, 75-year-old male who suffered an ischemic stroke 10 months prior to the study. He presented with right hemiparesis and a mild kyphosis because of posture- and age-related musculoskeletal changes. Passive shoulder flexion was limited to $140^{\circ}$, abduction to $90^{\circ}$ and external rotation to $30^{\circ}$. Proximal weakness at the shoulder girdle limited active shoulder abduction to $80^{\circ}$, and flexion to $100^{\circ}$ was observed, but with compensatory patterns of excessive shoulder elevation and abduction. He had full elbow ROM but was limited to $15^{\circ}$ of supination, and also limited in end-range extension at the wrist with fingers fully extended because of flexor muscle tightness. He also presented with decreased fine motor and in-hand manipulation skills related to intrinsic hand weakness, making tasks like using a fork very difficult. Neither subject presented with visuospatial neglect.

A 27-year-old, right-hand-dominant, healthy male participant followed the same testing procedures as the stroke participants.

\section{Data Analysis}

The FEATHERS Motion application was used to capture the position data and the clicking and visual state from both controllers at a frequency of $\sim 70 \mathrm{~Hz}$. The clicking state indicated if the controllers' left or right buttons were pressed, and the visual state was used as a flag to indicate if the controllers were out of the field of view of the camera. Data were stored in a binary file and later converted to a Microsoft ${ }^{\circledR}$ Excel format using a custom MATLAB ${ }^{\circledR}$ (Mathworks, Inc.) script. Video recordings were used to isolate the data during VS Mode play. Game session data were trimmed to the shortest playtime (2.7 minutes) common to the three participants. 


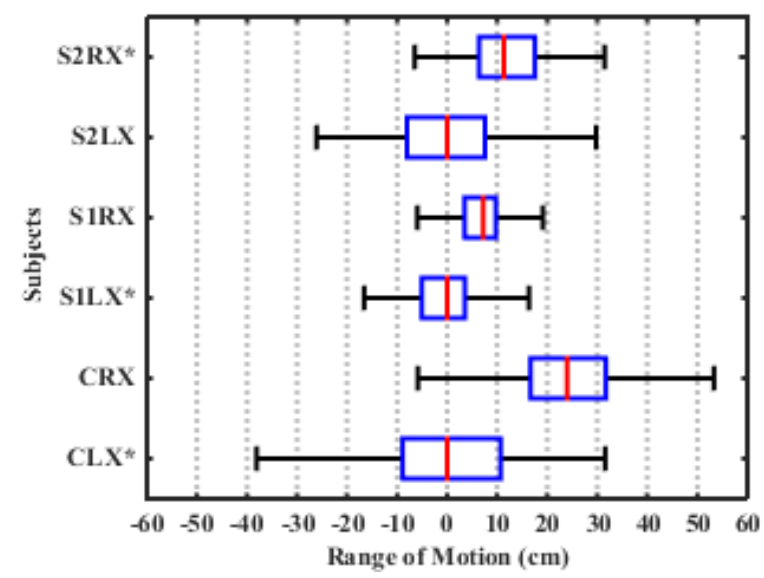

Fig. 3. ROM in the $\mathrm{x}$-direction. $\mathrm{R}=$ right hand; $\mathrm{L}=$ left hand; *=paretic hand (stroke participant) or non-dominant hand (control).

Data filtering was first applied to remove the controller data that were occluded or out of the camera's field of view, as indicated by the visual state in the log files. Given that the sampling frequency was not constant for our system, the data was linearly interpolated and resampled to a frequency of $50 \mathrm{~Hz}$. The data was then low pass filtered at $6 \mathrm{~Hz}$ [9] with a fourthorder zero-lag Butterworth filter. Both the interpolation and low pass filter were applied using a custom MATLAB script. Velocity and acceleration data were calculated by differentiating the resampled and filtered position data using a finite difference method.

The active ROM that users exhibited for each hand during the gaming session was calculated as follows. The 3D coordinate system was defined by the positive $\mathrm{X}$ direction to the right of the person, the positive $\mathrm{Y}$ direction vertically upwards, and the positive $\mathrm{Z}$ direction away from the camera. Because the game only required frontal plane movements to move the computer cursor on the screen, the analysis included only the $\mathrm{X}$ and $Y$ coordinates of the hand position. In addition, for this pilot study, the participants were free to choose a comfortable arm posture where $Z$ stayed approximately constant. Nevertheless, the $\mathrm{Z}$ direction could be used for future interventions and analyses in which a game might require sagittal plane movements.

Analysis of the $\mathrm{X}$ and $\mathrm{Y}$ movements of each hand provided the medians, quartiles and ranges for each subject's data set. Boxplots (Fig. 3 and Fig .4) were then created to visualize the differences between the subjects' hand offsets and the exhibited ROM. In both figures, the median of the left hand of each subject was subtracted from both hands to continually retain the left hand median at the zero position. This adjustment allowed for ease of visual comparison of the offsets and ROM between and within subjects.

Movement distribution analysis of the game play data was performed to create density plots of each participant's motion in the XY plane. These plots represent the natural way each participant distributes his or her motion in each of the position, velocity, and acceleration domains. From the collected data, histograms of left and right hand positions, velocities, and accelerations were calculated. These histograms were then

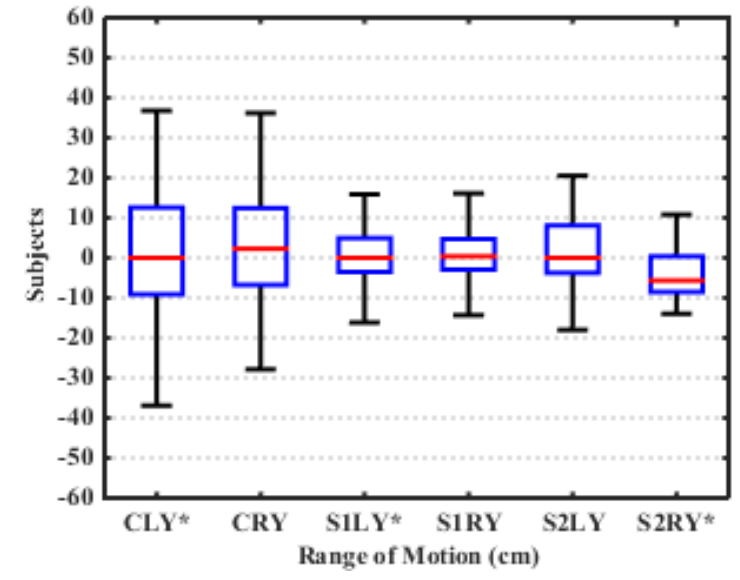

Fig. 4. ROM in the $\mathrm{y}$-direction. $\mathrm{R}=$ right hand; $\mathrm{L}=$ left hand; *=paretic hand (stroke participant) or non-dominant hand (control).

converted to density plots by using contour lines to show the natural and preferred behaviour of each participant. Our study builds on the work of Lancaster [10], Huang [11], and Fisher [12] who have shown correlations between stroke-specific impairments and a person's motion distributions. These studies have mainly concentrated on statistical modeling of the motion probabilities in different domains. In this paper, we present how summarizing such motion distributions in density plots can help therapists gain better insight into the impairments and motion tendencies of their clients.

\section{RESULTS AND DISCUSSION}

\section{A. Range of Motion}

The ROM for the X and Y directions are presented in Fig. 3 and Fig. 4, respectively. The whiskers of the boxplots extend to the upper (UAV) and lower adjacent (LAV) values, using 1.5 times the interquartile range as the limit for these values.

For both the $\mathrm{X}$ and $\mathrm{Y}$ directions, the ROM of the control participant was larger than those of the stroke participants. This result was expected based on the motor limitations of the stroke participants. Normative ROM values may be valuable for therapists from a rehabilitation perspective as a comparative benchmark for clients as they progress. Future phases of this project will include a larger sample of healthy controls for this purpose.

From the difference between the ROM medians of the two hands we can observe the typical offset that a user maintained during game play. For S2 the offset in $\mathrm{Y}$ showed that the impaired (right) hand was below the position of the unimpaired one most of the time. Video footage confirmed this data, as the user kept his affected hand close to his thigh instead of lifting it against gravity as on his unaffected side, as shown in Fig. 5, right. The offset in X and Y for S1 was smaller than the other participants' data, which suggests that $\mathrm{S} 1$ kept her hands closer together during game play. This inference was confirmed on review of the video, in which the participant compensated for weakness by placing her hands in contact with one another as she moved the controllers (Fig. 5, left).

Previous focus group [13], [14] and usability testing [6] studies of the FEATHERS project identified the need to monitor 


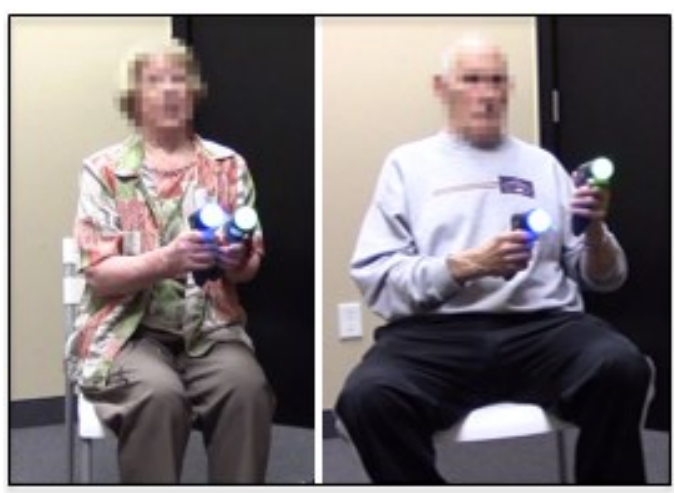

Fig. 5. S1 (left) and S2 (right) during game play

compensatory strategies during unsupervised rehabilitation (e.g., maintaining contact between the hands to create a point of stability for the unaffected hand, in order to enhance accuracy and control, and consequently, performance success). To mitigate this issue in instances where therapeutic goals call for independent hand movements, two offset parameters were included in the FEATHERS Motion application; a minimum X offset and a maximum $\mathrm{Y}$ offset. These parameters enable therapists to personalize the distance they want their clients to maintain between hands during play. This feature enables the ongoing customization of the therapy program as clients show improvements in motor control.

\section{B. Density Plots}

In this section we present density plots of motion distributions in position (Fig. 6), velocity (Fig.7), and acceleration (Fig. 8) subspaces for the three participants. The contours in Fig. 6 are colour-coded to show the minimum observed time spent at each point in a $90 \mathrm{~cm} \times 90 \mathrm{~cm}$ (using a $2.5 \mathrm{~cm} \times 2.5 \mathrm{~cm}$ grid) workspace in the frontal plane. In the plots presented in Fig. 6, the points that are surrounded by a contour line have appeared in the position data for at least the amount of time that corresponds with the colour of the contour line. The origin of the workspace was set for each participant to the lowest leftmost point that his/her left hand visited during game play.

As evident in the plots of Fig. 6, and as presented in Fig. 3 and Fig. 4, the healthy participant $\mathrm{C} 1$ (top row) has a much larger active ROM than S1 and S2 (bottom row) by a ratio close to 3:1. A more compelling finding from the density plots of the position distributions is the location uniformity that the healthy participant demonstrates. The dominant colour in the plots of the healthy participant is navy blue (i.e., less than $1 \mathrm{sec}$ at each location) and there is no trace of a colour outside the blue colour family. S1 and S2 have smaller ROM and spent on average more time at each location. This finding is highlighted by the dominant cyan/teal colour of their position density plots, which maps to $2 \mathrm{sec}$ at each location. In contrast to $\mathrm{C} 1$, the two stroke participants' position density plots exhibit a wide range colours (i.e., wide range of values compared to $\mathrm{C} 1$ ), indicating that they do not equally distribute time spent at each location within their active ROM. The nature of this unequal distribution pattern and the location of the hot spots (i.e., red contours) has been used previously to identify motor deficits [10], [11], which in turn may inform therapy for an individual.
S2 kept his hands separated and did not use compensatory stabilizing strategies. The affected right hand's motion is not equally distributed (as evidenced by the range of colours and hot spots in the plot), whereas the left hand exhibits a more equal distribution pattern (compare colours with $\mathrm{C} 1$ ). His smaller ROM compared to $\mathrm{C} 1$ on his healthy (left) side may be related to the fact that the motion of the mouse cursor in the game was mapped to the motion of his weaker right side. As using his entire ROM of the left hand would not lead to a bigger cursor motion, it is possible that he matched the ROM of his strong side to his weaker side to save energy. In contrast, S1 kept her hands close together as she played the game, as evidenced by similar position density plots of her hands, as well as by video review findings. This strategy may have helped her perform the bimanual task by enabling her strong side to compensate for the weakness in her other arm.

The contours in Fig. 7 mark areas with the same number of occurrences, calculated as the percentage of the total observations. The hand velocity was calculated by differentiating position data using a finite difference method. The histogram data of velocity within a $80 \mathrm{~cm} / \mathrm{s} \times 80 \mathrm{~cm} / \mathrm{s}$ area was generated using a $4 \mathrm{~cm} / \mathrm{s} \times 4 \mathrm{~cm} / \mathrm{s}$ grid and then plotted with a contour format.

Similar to position density plots of Fig. 6, existence of a range of colours and hot spots in the velocity plots of Fig. 7 suggests an uneven distribution of motion, which may be related the motor deficits of an individual; further examination of this relationship is warranted. Also, noting the minor difference in the shape and colours of the left and right hand plots (e.g., for S1) may provide a basis for monitoring the use of bimanual stabilizing compensatory strategies by users with the tendency to use them, although further exploration with a larger sample would be needed to confirm the utility of this analysis strategy. $\mathrm{C} 1$ shows a velocity contour profile that is close to a diamond shape. In general, $\mathrm{C} 1$ created higher velocities compared to S1 and S2. Density plots may be used in this way to monitor speed of movement in different directions as a therapy outcome.

The contours in Fig. 8 mark areas with the same number of occurrences, calculated as the percentage of the total observations. Similar to hand velocity, hand acceleration was also calculated by differentiating using a finite difference method. The histogram data of acceleration within a $600 \mathrm{~cm} / \mathrm{s}^{2}$ $\times 600 \mathrm{~cm} / \mathrm{s}^{2}$ area was generated using a $30 \mathrm{~cm} / \mathrm{s}^{2} \times 30 \mathrm{~cm} / \mathrm{s}^{2}$ grid and then plotted in a contour format.

Similar to the position and velocity density plots, the $\mathrm{C} 1$ acceleration plot is more evenly distributed (low percentage of occurrences on the displayed contours) than for S1 and S2. Moreover, S1 and S2 have smaller maximum accelerations compared to $\mathrm{C} 1$, which suggests that the healthy subject was able to produce larger forces with his arms in all directions when compared to the stroke participants. In addition, both stroke participants spent most of the time moving with smaller accelerations as indicated by the red/orange contours that are close to zero. We hypothesise that the shape of the acceleration contour profile could be considered as an indicator of the ability to produce larger forces in different directions during the progress of a rehabilitation program. 

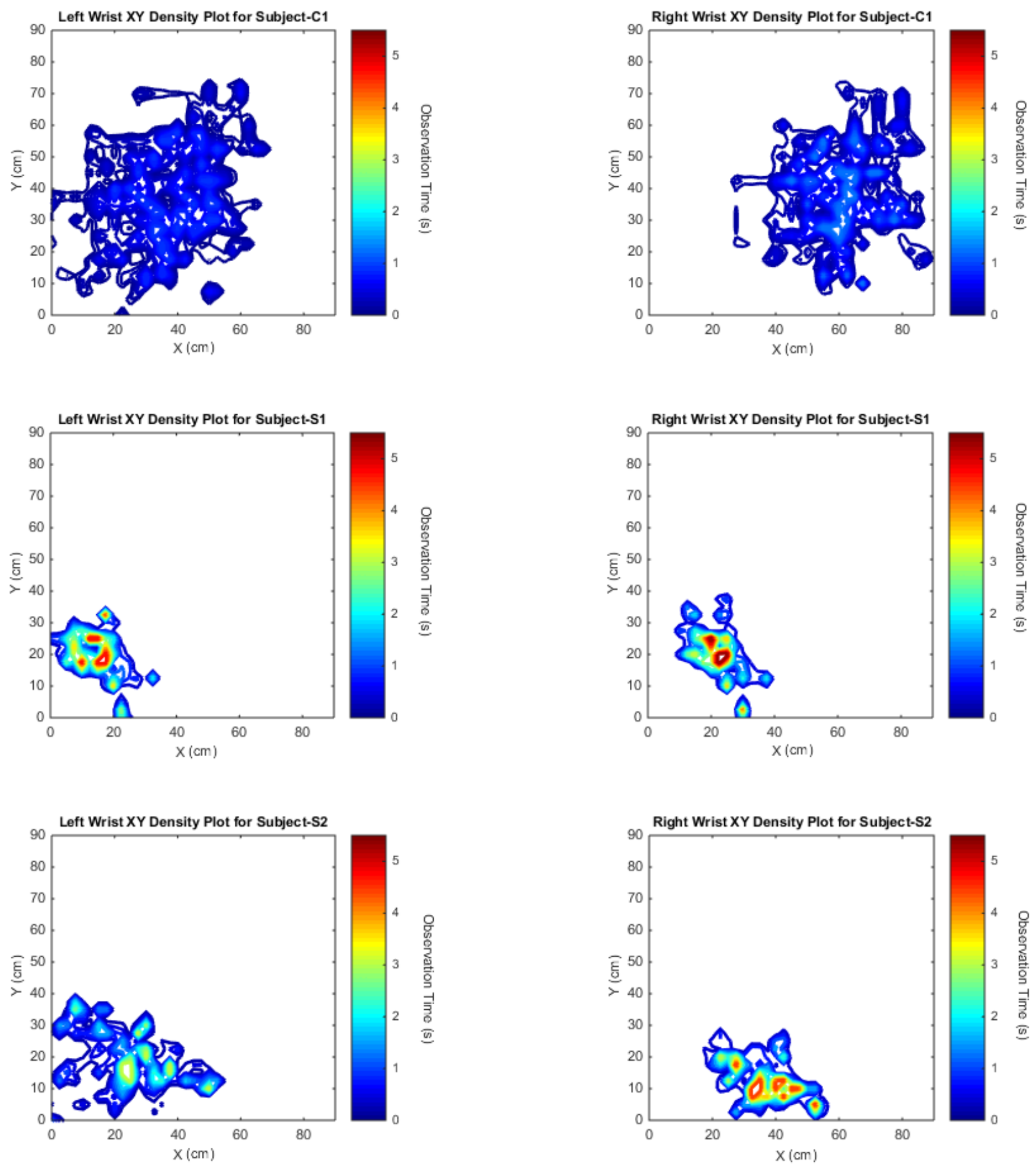

Fig. 6. Density plots of the left and right hand positions of each participant. C1 was a healthy adult and S1 (left side weakness) and S2 (right side weakness) were stroke survivors. Contour lines summarize the minimum observed time of the area they surround in the collected position data.

\section{CONCLUSIONS}

The FEATHERS Project has implemented a novel bimanual control modality to encourage persons with hemiplegia to use their more-affected side in relative symmetry with their lessaffected side to control a cursor in a computer game. This algorithm is designed to promote bimanual upper limb movements and to allow for their quantification. Kinematic analysis of upper limb motion can now include not only absolute ROM progression over time in multiple directions ( $\mathrm{X}$ and $\mathrm{Y}$ in this case), but also relative movement parameters of the two arms engaged in a single task. The analysis has led to several different and anatomically/neurologically-relevant visualizations of task space coverage in position, velocity and acceleration, and has shown clear differences between one healthy control and two post-stroke participants. Functional and 

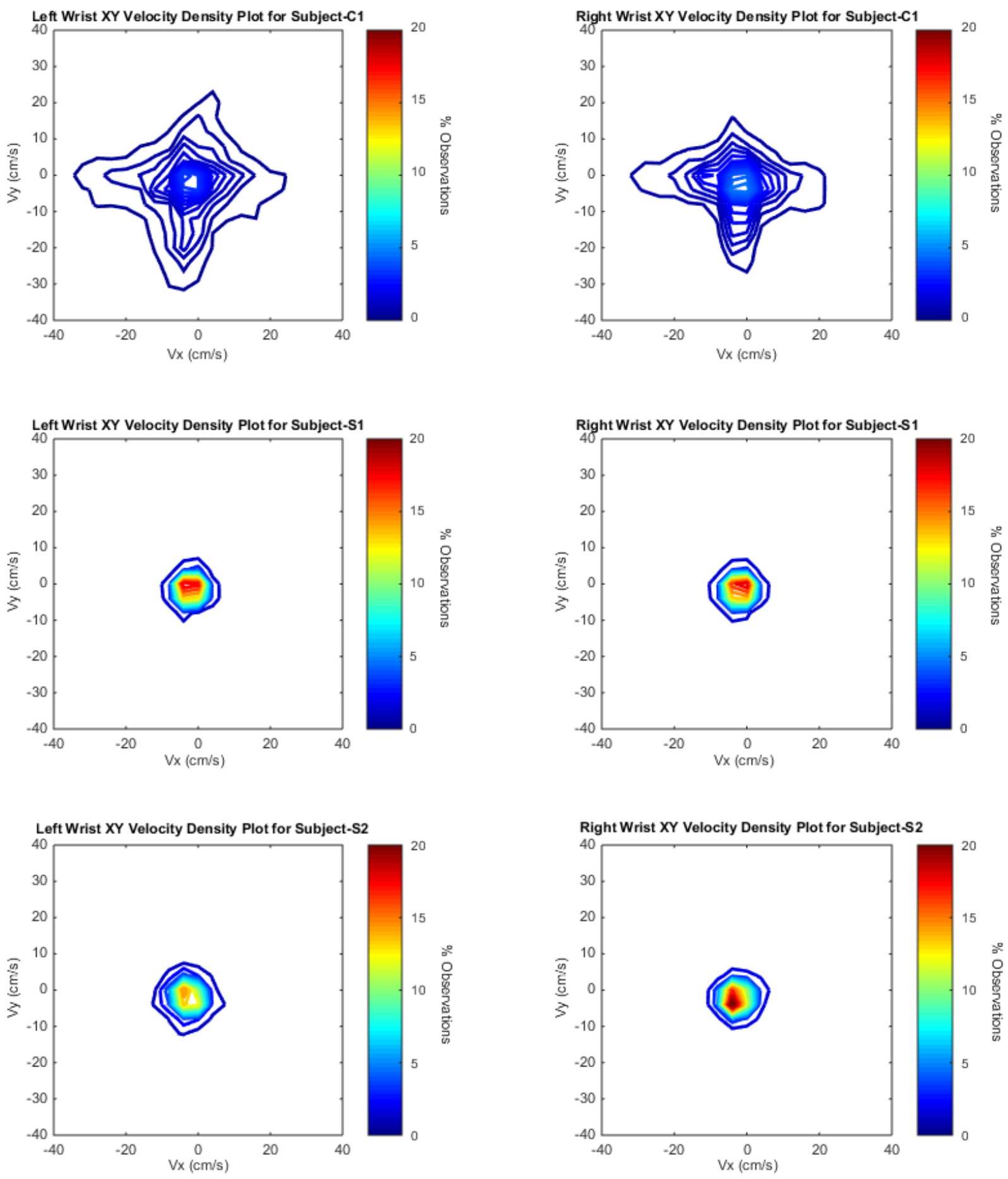

Fig. 7. Density plots of the observed velocities of the left and right wrists for each participant. C1 was a healthy adult and S1 (left side weakness) and S2 (right side weakness) were stroke survivors. Contour lines are color-coded to mark the percentage of the total observations.

spatial differences can be described numerically and graphically, providing therapists with the ability to track performance changes over time, and to evaluate the movementspecific and directional differences demonstrated by their clients over time.

For this pilot study, only the data from three subjects were employed. However, the next phase of the project will provide the opportunity to apply the different representation techniques to a larger sample with comparison to clinical evaluation of function and outcomes in order to enable an assessment of sensitivity and effectiveness of the system. During this final, inhome testing phase, therapists will receive weekly reports on their clients' ROM, hand velocity, as well as time played and distance travelled by each hand. This information will be used to monitor the progress of clients' rehabilitation programs and 

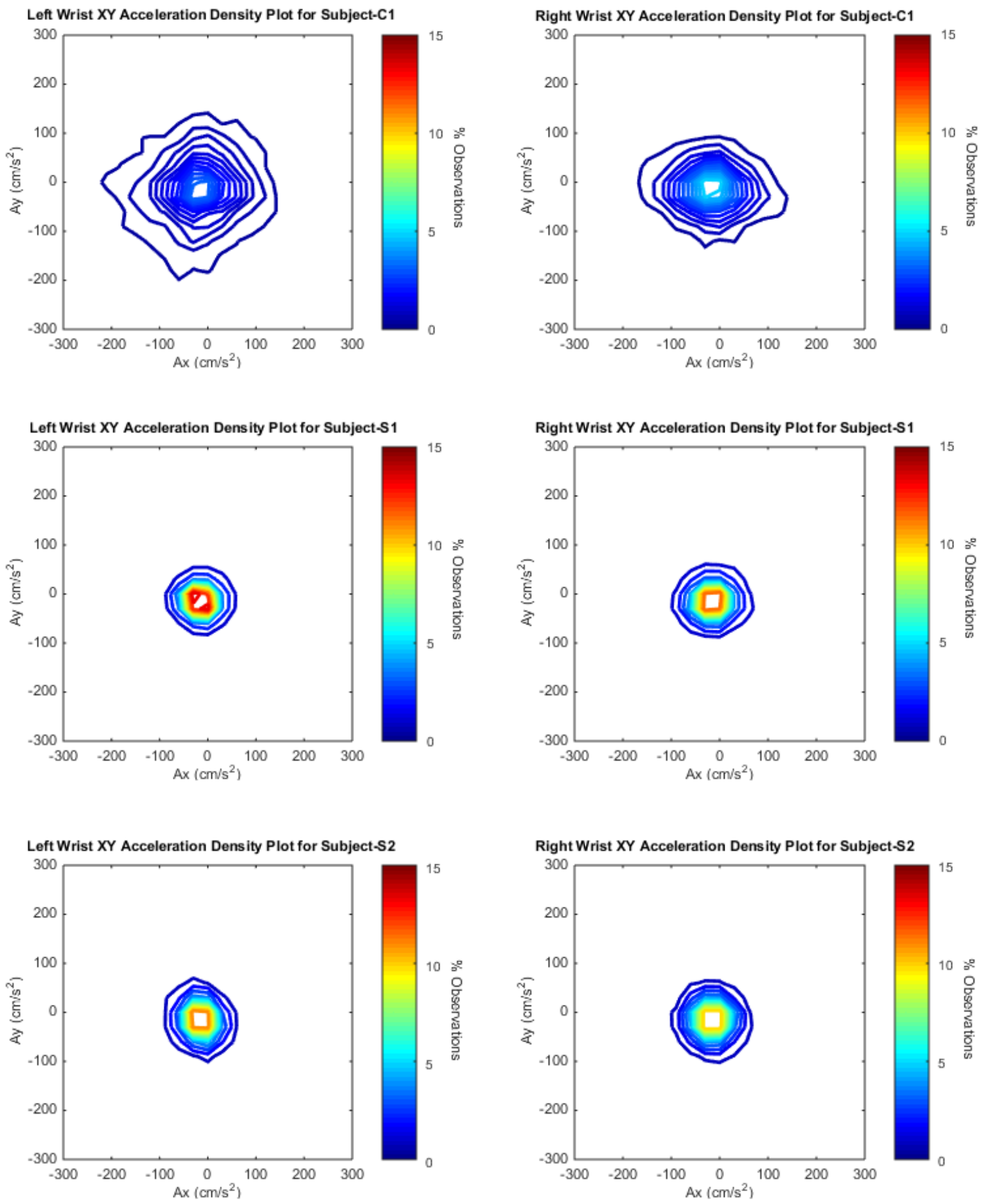

Fig. 8. Density plots of the observed accelerations of the left and right wrists for each participant. C1 was a healthy adult and S1 (left side weakness) and S2 (right side weakness) were stroke survivors. Contour lines are color-coded to mark the percentage of total observations.

to allow therapists to provide reinforcing feedback about functional performance status and improvements over time.

\section{ACKNOWLEDGMENT}

We would like to acknowledge Abilities Neurological Rehabilitation (Vancouver, Canada), the software company Reality Controls Inc. (Vancouver, Canada), student intern Brandon Kim, the FEATHERS team and the three participants.

\section{REFERENCES}

[1] K. Thomson, A. Pollock, C. Bugge, and M. Brady, "Commercial gaming devices for stroke upper limb rehabilitation: a systematic review.," Int. J. Stroke, vol. 9, no. 4, pp. 479-88, Jun. 2014.

[2] E. Pietrzak, C. Cotea, and S. Pullman, "Using commercial video games for upper limb stroke rehabilitation: is this the way of the future?," Top. Stroke Rehabil., vol. 21, no. 2, pp. 152-162, 2014.

[3] K. R. Lohse, C. G. E. Hilderman, K. L. Cheung, S. Tatla, and H. F. M. Van der Loos, "Virtual reality therapy for adults post-stroke: a 
systematic review and meta-analysis exploring virtual environments and commercial games in therapy.," PLoS One, vol. 9, no. 3, p. e93318, Jan. 2014.

[4] M. King and J. Hijmans, "Home-based stroke rehabilitation using computer gaming," New Zeal. J. Physiother., vol. 40, no. 3, pp. 129$135,2012$.

[5] D. Webster and O. Celik, "Systematic review of Kinect applications in elderly care and stroke rehabilitation," J. Neuroeng. Rehabil., vol. 11, no. 1, p. 108, 2014.

[6] B. A. Valdés, C. G. E. Hilderman, C. T. Hung, N. Shirzad, and H. F. M. Van der Loos, "Usability testing of gaming and social media applications for stroke and cerebral palsy upper limb rehabilitation," in 36th Annual International Conference of the IEEE Engineering in Medicine and Biology Society (EMBC), 2014, pp. 3602-3605.

[7] S. M. N. Glegg, C. T. Hung, B. A. Valdés, B. D. G. Kim, and H. F. M. Van der Loos, "Kinecting the moves: the kinematic potential of rehabilitation-specific gaming to inform treatment for hemiplegia," in 10th Intl . Conf. Disability, Virtual Reality \& Associated Technologies, 2014, pp. 313-316.

[8] N. Shirzad, B. A. Valdés, C. Hung, M. Law, J. Hay, and H. F. M. Van der Loos, "FEATHERS, a bimanual upper limb rehabilitation platform: a case study of user-centred approach in rehabilitation device design," in International Conference on Engineering Design ICED 2015, 2015, p. to appear.

[9] R. M. Enoka, Neuromechanics of Human Movement. Human Kinetics, 2008.

[10] J. Lancaster, Z. Wright, F. Huang, and J. L. Patton, "The Effects of Motor Impairments on Movement Distributions," in 36th Annual International Conference of the IEEE Engineering in Medicine and Biology Society (EMBC), 2014.

[11] F. Huang and J. Patton, "Individual patterns of motor deficits evident in movement distribution analysis.," in 2013 IEEE International Conference on Rehabilitation Robotics (ICORR), 2013, pp. 1-6.

[12] M. Fisher, F. Huang, Z. Wright, and J. Patton, "Distributions in the error space: Goal-directed movements described in time and state-space representations," in 36th Annual International Conference of the IEEEEngineering in Medicine and Biology Society (EMBC), 2014, pp. 6953-6956.

[13] M. Lam, S. Tatla, K. Lohse, N. Shirzad, A. Hoens, K. Miller, N. VirjiBabul, and M. Van der Loos, "Perceptions of Technology and Its Use for Therapeutic Application for Individuals With Hemiparesis: Findings From Adult and Pediatric Focus Groups," JMIR Rehabil Assist Technol, vol. 2, no. 1, 2015.

[14] S. Tatla, N. Shirzad, K. Lohse, N. Virji-Babul, A. Hoens, L. Holsti, L. Li, M. Lam, and M. Van der Loos, "Therapists' Perceptions of Social Media and Video Game Technologies in Upper Limb Rehabilitation," JMIR Serious Games, vol. 3, no. 1, 2015.

(C) 2015 IEEE. Personal use of this material is permitted. Permission from IEEE must be obtained for all other uses, in any current or future media, including reprinting/republishing this material for advertising or promotional purposes, creating new collective works, for resale or redistribution to servers or lists, or reuse of any copyrighted component of this work in other works. 